\title{
In vivo testing of a bone graft containing chitosan, calcium sulfate and osteoblasts in a paste form in a critical size defect model in rats
}

\author{
Jerome G. Saltarrelli Jr. ${ }^{1}$, Debi P. Mukherjee ${ }^{1 *}$ \\ Louisiana State University Health Sciences Center Department of Orthopaedic Surgery, 1501 Kings Highway, Shreveport, Louisiana 71130, * Corresponding \\ author to Debi P. Mukherjee: (dmukhe@1suhsc.edu) \\ Received September $26^{\text {th }}, 2008$; revised November $12^{\text {th }}, 2008$; accepted November $13^{\text {th }}, 2008$
}

\begin{abstract}
Bone loss associated with musculoskeletal trauma or metabolic diseases often require bone grafting. The supply of allograft and autograft bones is limited. Hence, development of synthetic bone grafting materials is an active area of research. Chitosan, extracted from chitin present in crawfish shells, was tested as a delivery vehicle for osteoblasts in a 2-3 $\mathrm{mm}$ size defect model in rats. Twenty-seven male Lewis rats, divided into three groups with sacrifice intervals of 3, $6 \& 9$ months were used. In the experimental samples, a critical size defect was filled with chitosan bone graft paste and fixed with a plate, while in the operated control group, a critical size defect was repaired only by a plate (no paste was applied). An unoperated control group was also included. Bone growth was evaluated histologically by examining undecalcified and decalcified stained sections. The femurs were also examined non-destructively by micro-computed tomography $(\mu \mathrm{CT})$. Defects filled with chitosan bone graft paste demonstrated superior healing across all time periods compared to unfilled defects as examined by histology and micro-computed tomography. Crawfish chitosan has successfully been used as a cell delivery system for osteoblasts for use as a synthetic bone graft material.
\end{abstract}

Keywords: Chitosan, Synthetic Bone Graft, Cell Delivery, Histology, Animal Model Running Head: Chitosan Based Synthetic Bone Graft Material

\section{INTRODUCTION}

Bone loss associated with musculoskeletal trauma or metabolic diseases often require bone grafting. Autograft or allograft bones are limited in supply. Therefore, development of synthetic bone graft materials is an active area of research.
Chitin is a polysaccharide that exists in fungi, exoskeleton of insects and the outer shell of crustaceans $[1,2,3]$. It is biocompatible [4], osteoconductive [5], antimicrobial [6,7], biodegradable [8], non-toxic [9], haemostatic [10], fungicidal [11], and the second most abundant natural polysaccharide on earth [12]. Removal of the acetyl group from chitin forms chitosan. Chitosan is more useful due to the presence of amino groups that impart a positive charge to the molecule. Chitosan has been investigated in a number of biomedical applications due to its purity coupled with a positive charge $[13,14,15,16]$. This positive charge interacts with cells or can act as a binding site for other functional groups thereby expanding the role of the chitosan molecules.

Recently, a new patented process has been developed to purify chitosan from crawfish shells [17]. The objective of this current study was to evaluate this crawfish chitosan as a delivery system for osteoblasts to promote bone growth in a $2-3 \mathrm{~mm}$ defect in rats. For this purpose, prepared crawfish chitosan was compounded with calcium sulfate to form a paste. The bone growths at 3, $6 \&$ 9 months were estimated by histology and microcomputed tomography $(\mu \mathrm{CT})$. It was hypothesized that chitosan provides a delivery system, keeping the cells in the defect area for a longer period of time allowing defect repair.

\section{MATERIALS AND METHODS}

\subsection{Chitosan Extraction}

Chitosan was extracted from crawfish shells following a patented process [17]. Briefly, shells were first washed, and dried in an $80^{\circ} \mathrm{C}$ oven for 48 hours. Following drying, the shells were immediately quenched in liquid nitrogen then treated by: (1) $3.5 \% \mathrm{NaOH}$ to remove proteins; (2) $1 \mathrm{~N} \mathrm{HCl}$ to remove minerals; and (3) $50 \%$ $\mathrm{NaOH}$ to remove acetyl groups. The extracted chitosan was purified through a $12,000-14,000$ Dalton dialysis membrane and dried.

\subsection{Preparation of Osteoblasts}

Stromal osteoblast cells were obtained from the marrow 
of young adult male (125-149g) Lewis rats. Following euthanasia by $\mathrm{CO}_{2}$ asphyxiation, femora were aseptically excised, cleaned of soft tissue, washed in DMEM+ antibiotic-antimycotic (concentration of antibiotic- antimycotic was 10 times the normal amount used in cellular media). The metaphyseal ends were cut off and the marrow flushed from the midshaft with $5 \mathrm{ml}$ of media (DMEM+10\% FBS+antibiotic-antimyctotic) using a syringe equipped with a 22-gauge needle and collected in a sterile test tube. Cell clumps were broken up by repeated pipetting of the cell suspension. The cells were centrifuged at $1200 \mathrm{rpm}$ for 10 minutes. The cell pellet was resuspended in media (DMEM+10\% FBS + antibiotic-antimycotic $+10 \%$ IL-3) and seeded in a flask. On the following day, the media was removed and the cells were washed with $10 x$ concentration of antibiotic- antimycotic in PBS; and complete media was introduced into the flask. Cells were fed every two to three days until confluent, and then trypsinized.

\subsection{Paste Formulation}

The ratio of chitosan to calcium sulfate was $1: 4$, ideally $0.125 \mathrm{~g}$ of crawfish chitosan to $0.5 \mathrm{~g}$ calcium sulfate. The actual average paste contents were $0.5030 \mathrm{~g}$ of calcium sulfate and $0.1234 \mathrm{~g}$ of crawfish chitosan with an average osteoblast concentration of $4.06 \times 10^{5} / \mathrm{ml}$. The calcium sulfate $\left(\mathrm{CaSO}_{4}\right)$ and extracted chitosan, after sterilization under dry heat, was mixed with $1 \mathrm{ml}$ of osteoblast cell suspension $\left(10^{6}\right.$ cells $\left./ \mathrm{ml}\right)$ to form a paste. This was accomplished immediately prior to implantation under the sterile field. The same procedure was used in a previous study repairing a cranial critical size defect in rats [18].

\subsection{Experimental Design and Animal Sur- gical Procedure}

Twenty seven male Lewis rats were divided into 3 groups: (1) operated control (2) experimental and (3) non-operated control. Animals in the operated control and experimental groups went through a surgical procedure which created a segmental defect of $2-3 \mathrm{~mm}$ which is a modification of the $8 \mathrm{~mm}$ critical size segmental defect model [19,20]. This modification was necessary because a four-hole $23 \mathrm{~mm}$ long plate was used, which had a solid section of about $4 \mathrm{~mm}$ between the second and third hole covering the femoral defect. This modification in defect size was necessary for proper femoral repair. The operated control group animals received only plate fixation. The experimental group received both plate fixation and bone graft paste. The unoperated group was a control. Each group, of three animals per time period, was studied for 3, 6 and 9 months.

The surgical procedure is as follows: animals were anesthetized with $1.5 \%$ Isoflurane, shaved and cleaned with $70 \%$ alcohol and Betadine. An incision was made dorsally to the femur and a 4-hole titanium plate was applied. In both operated groups (experimental and operated control), once the plate was installed a $2-3 \mathrm{~mm}$ size defect was applied to the femur using a burring bit on a Dremel tool. In the operated control group, the incision was closed. In the experimental group, the chitosan-bone graft paste was applied to the defect. Post surgery, animals were housed in individual cages and monitored for surgical complications (foot drop, lethargy, loss of use and pain). Upon euthanizing by $\mathrm{CO}_{2}$ asphyxiation, operated and contra-lateral control femurs were removed and stored frozen until testing in a $0.9 \%$ $\mathrm{NaCl}$ solution.

\subsection{Undecalcified Histological Sample Preparation and Staining}

All undecalcified histological samples were fixed in $10 \%$ formalin for at least 2 weeks. Following formalin fixation, the samples were taken through an increasing gradient of 2-hydroxyethyl-methacrylate and nanopure water then a transition of 2-hydroxyethyl-methacrylate and Technovit 7200 and finally $100 \%$ Technovit 7200 . Samples were embedded in resin by submersion in fresh Technovit 7200 and exposure to an ultraviolet light $(\lambda=450 \mathrm{~nm})$. Ultraviolet light causes polymerization of Technovit 7200. Sample blocks were attached to slides using Technovit 7210LVC, cut using an Exakt band saw and ground into $10-20 \mu \mathrm{m}$ thick slices using an Exakt surface grinder (Exakt Technologies, Oklahoma City, OK).

All undecalcified histological sections were stained using Goldner's trichrome method whereas bone stains green, muscle stains orange, and fibrous tissue stains red.

\subsection{Non-destructive Evaluation by Micro- computed Tomography $(\mu \mathrm{CT})$}

$\mu \mathrm{CT}$ was preformed (courtesy of Animal Resources, Louisiana State University Health Sciences Center, Shreveport, Louisiana) on a MicroCAT II; model MCIIUAAI following standard operating procedure. Each femur, along with the contra-lateral side, was run for 512 scans along the defect site. This data was compiled into 2-D and 3-D images and qualitative information on bone growth was collected.

\section{RESULTS}

\subsection{Undecalcified Histological Sectioning}

Three, six and nine months data for operated control and experimental samples are shown in Figure 1, A through I and an example of unoperated mature bone is shown in Figure 1-J. Goldner's Trichome stain was used which stains bone green and muscle and fibrous tissues red. All photographs shown were taken at $4 \mathrm{x}$ magnification under a light microscope.

\subsection{Histological Quantitative Data}

From bone pixelation, bone percentages from each group can be compared (Figure 2). In each time interval, more bone was present in the experimental group compared to 


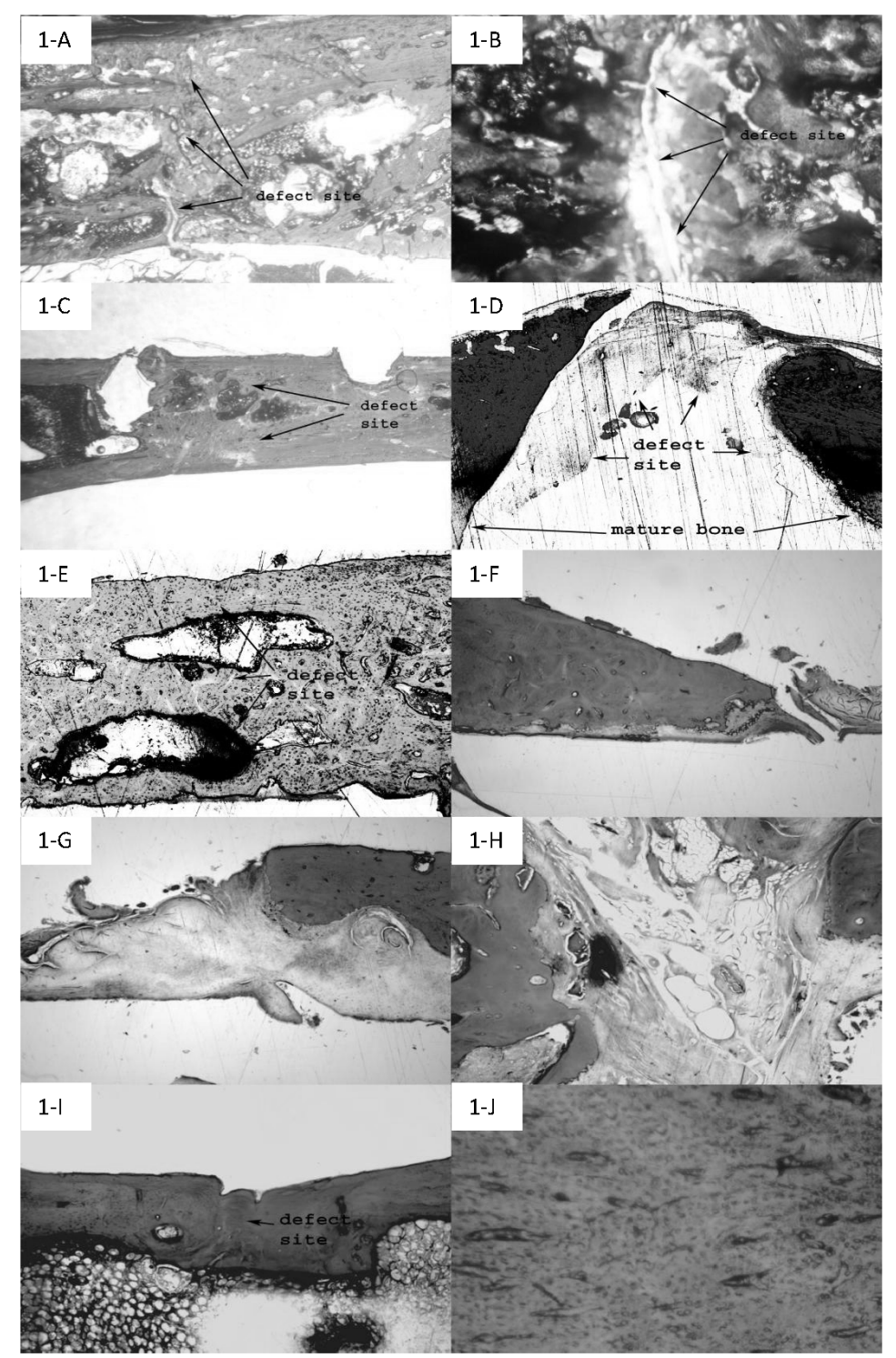

Figure 1. Undecalcified Histology. (Figure 1-A \& 1-B shows the presence of a defect after 3 months without chitosan/ Plaster of Paris bone graft paste (operated control group) (1-A=5x; 1-B=20x). Figure 1-C shows experimental samples after 3 months, the defect was repaired with beginning of intermedullary canal. After 6 months, significant fibrous growth remained in the operated control group (Figure 1-D $=20 x$ ). In the 6 month experimental group, rapid repair has occurred (vs. operated control) with the presence of large voids (Figure $1-E=20 x$ ). Figure $1-F, G \& H(20 x)$ show the 9 month operated control group with infiltration of fibrous tissue which inhibits bone growth. Figure 1-I (5x) is the experimental group after 9 months with near complete bone growth. Figure $1-\mathrm{J}$ is an unoperated control (20x).)

the operated control group. During the three month interval, $40.23 \%$ bone growth was calculated in the experimental group while only $29.43 \%$ was calculated in the operated control group. At the six month time interval, $35.58 \%$ bone growth was calculated in the experimental group and $30.09 \%$ bone growth was recorded in the operated control group. As expected, nine month time interval demonstrated the highest percentage of bone growth in both groups. The experimental group experienced $42.82 \%$ bone growth while the operated control group experienced $40.16 \%$. In all the sacrifice intervals the degree of bone growth was slightly higher for the experimental group than that of the operated con- trol group. After three months, the experimental samples demonstrated $26.85 \%$ more bone in the defect site than the operated control samples. After six months, the experimental samples demonstrated $15.42 \%$ more bone and after nine months, $6.2 \%$ more bone was evident in the defect site. In depth statistical analysis of the data was not possible due to limited number of animals.

\subsection{Non-destructive Testing by Microcom- puted Tomography $(\mu \mathrm{CT})$}

$\mu \mathrm{CT}$ was preformed on a MicroCAT II; model MCII-UAAI following standard operating procedure. Each femur, along with the contra-lateral side, was run 
for 512 scans along defect site. This data was compiled into 2-D and 3-D images. We were unable to extract the quantitative information like bone volume or connectivity density of trabecular bone from the $\mu \mathrm{CT}$ images since we did not possess the appropriate software. Therefore we qualitatively looked at the 3-D rendered $\mu \mathrm{CT}$ images for bone repair. The 3, 6 and 9 month data are shown in Figures 3A-3F while Figure 3-G displays unoperated control data. MicroCT data showed gradual bone growth even at the 3 month period as seen with the computerized volumetric rendering data (3-B). At six and

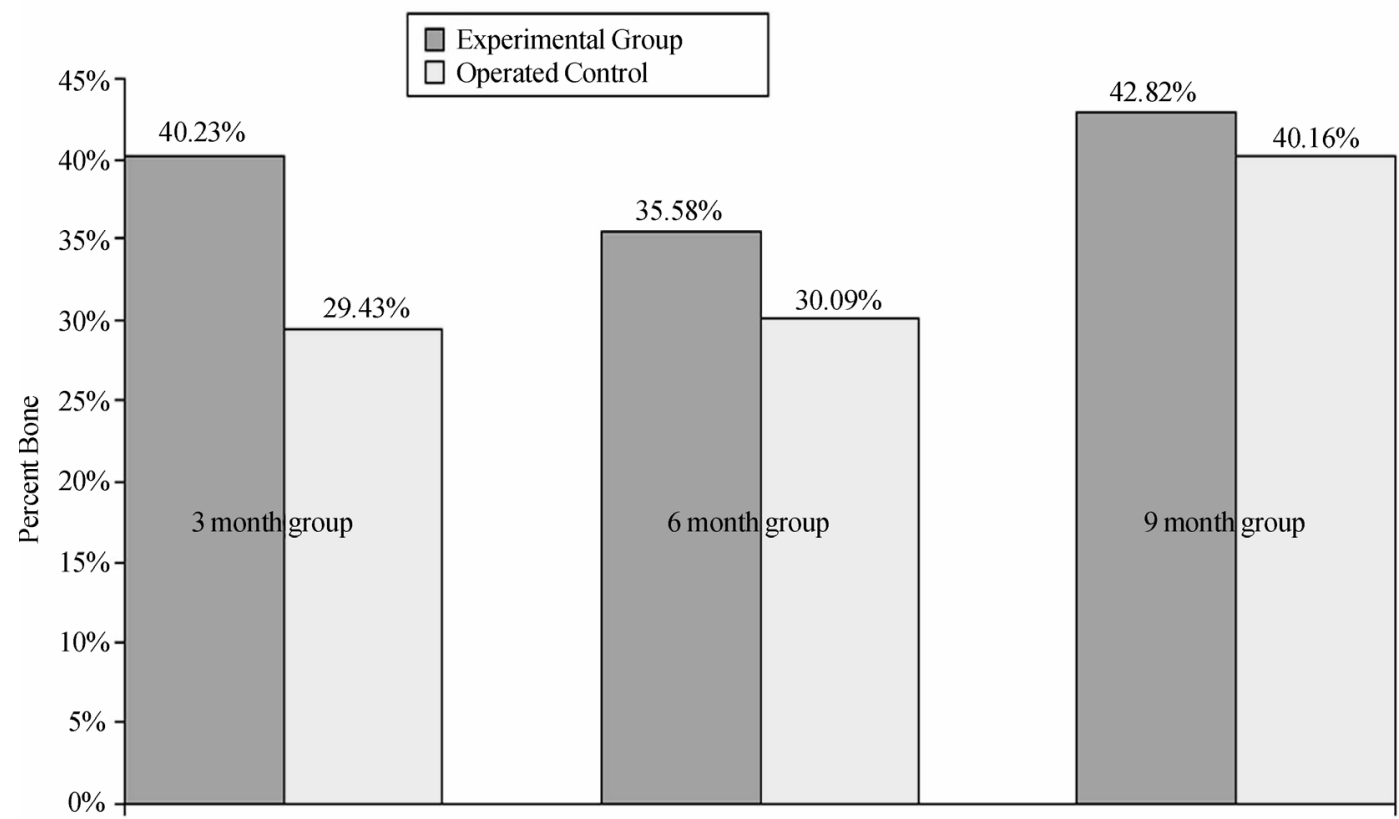

Figure 2. Bone Growth Fractions. (Bone growth fractions were calculated by pixel quantification within the defect site. In all time periods, more bone is present in the experimental samples versus the operated control samples, as represented here. Error bars represent variation between specimens.)

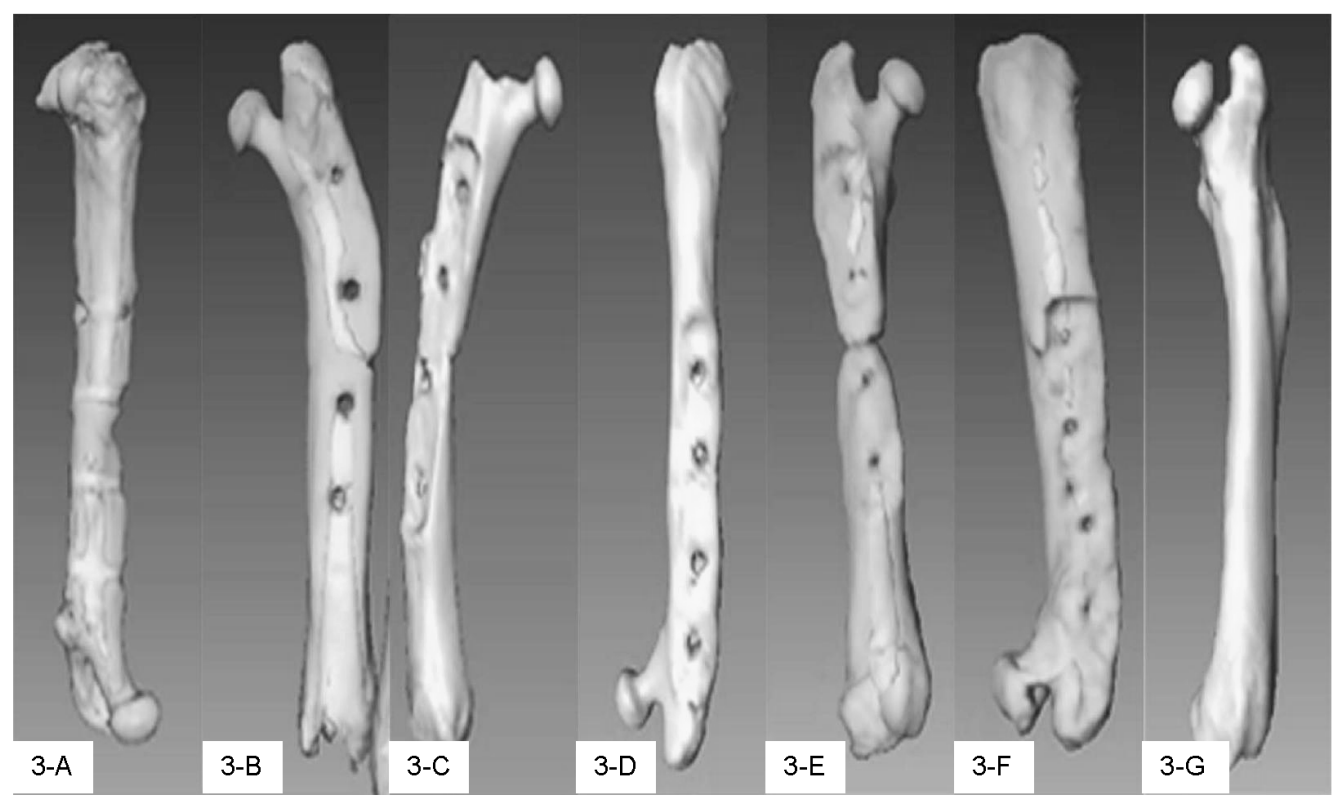

Figure 3. Microcomputed Tomography 3-D Images. (Figure 3-A is an operated control specimen after 3 months. An incomplete intermedullary canal as well as the presence of a surface defect can be visualized. Figure 3-B is an experimental sample after 3 months. Increase in the intermedullary canal and a decrease in the surface defect (versus the operated control sample from the same time point) can be seen. Figure $3-C$ is the operated control sample after 6 months. An apparent surface defect is present compared to the experimental sample (from the same time point) in figure 3-D. Figure 3-E and 3-F are the operated control and experimental samples, respectively, after 9 months. A complete defect, with boney ingrowth, can be seen in the operated control sample (Figure 3-E). Lack of significant surface defect can be visualized in the experimental sample (Figure 3-F) along with complete defect repair. Figure 3-G is an unoperated control.) 
nine months (Figures 3-D \& 3-F respectively) the defect-free bone is seen in the experimental group. However, the repair was incomplete in operated controls 3-A, 3-C and $3-\mathrm{E}$. This confirmed bone growth was stimulated in the experimental group.

\section{DISCUSSION}

\subsection{Undecalcified Histological Sectioning and Staining}

During each time interval (three, six and nine months) repair occurred faster in experimental groups compared to than in the operated control groups. The repaired area in the experimental group lacked fibrous tissue and, histologically, appeared to consist primarily of cortical bone. In comparison, operated control samples demonstrated repair with a relative high concentration of voids and fibrous tissues. Defects were present, in the operated control femurs, after even nine months of repair time.

The color variation in the sections was due to specimen thickness, whereby more tissue was stained. Using the Exakt system, exact specimen thickness is difficult to control. General colors were similar with slight shade variation. Exact duplication in slide thickness was attempted but not always achieved. Thus histological analysis demonstrated the presence of bone.

At the three month time interval, bone infiltrated into the defect site although infiltration was not complete, the defect could still be visualized. In operated control samples, incomplete healing began at the defect site. Bone infiltration was sporadic, filled with large voids, and the defect was still present (Figures 1-A \& 1-B). In experimental samples, the defect was completely filled with mature bone and the intermedullary canal was beginning to reform (Figure 1-C). In the operated control histological sections, repaired bone could be visualized, although the rate of repair was far inferior to repair seen in the experimental group.

Six month undecalcified histological operated control sections displayed significant fibrous growth at the defect site. Tissue juxtaposed to the defect site was highly infiltrated with fibrous tissue (Figure 1-D). Analysis of later sections revealed the area of fibrous tissue decreased in the defect site. Six month experimental histological sections displayed superior growth compared to the operated control samples. Repaired bone tissue displayed continuous growth although large voids, white spaces, were noticed (Figure 1-E). Six month experimental histological sections showed improved repair compared to operated control samples. While voids were present in experimental samples, operated control samples had complete defects present.

Nine month undecalcified operated control sections displayed a high relative percentage of fibrous tissue in the defect site. Unlike the six month group, fibrous tissue was juxtaposed to cortical bone (Figure 1-F). In the nine month undecalcified experimental sections, defects were only slightly present with no signs of fibrous tissue or voids (Figure 1-I). All defects, in experimental femurs, were repaired with, histologically, cortical bone. After surgery, rats gained weight due to lack of significant movement and therefore had less space for movement. This lack of significant movement reduced the mechanostimulation on all loaded skeletal structures, including the defected bone. Since repair processes strive under loaded conditions and these lethargic animals presented reduced loaded conditions, bone growth decreased from the three month group to the nine month group. During all time intervals, animals were confined to Institutional Animal Care and Use Committee (IACUC) approved cages 10.5" W x 19" L x 8" H (Allentown Caging Equipment, Inc., Allentown, N.J.), physical activity was negligible and feed intake was constant (free choice). Reduced physical activity led to decreased mechanostimulation which led to weakened repaired bone $[21,22]$. Nevertheless the nine month experimental group demonstrated a relative higher percentage of bone compared to operated control femurs from the same time period.

\subsection{Non-Destructive Testing by Microcom- puted Tomography $(\mu \mathrm{CT})$}

MicroCT $(\mu \mathrm{CT})$ is a series of $\mathrm{x}$-ray images compiled together into a three dimensional image. Individual slices were available for viewing as well as three dimensional images. Microcomputed tomography images of the bone graft site displayed progressive repair in experimental samples compared to operated control femurs, which displayed a complete defect throughout all time periods. Operated control femurs, across all time intervals, displayed similar characteristics. All operated control samples had incomplete intermedullary canals, defect presence (appropriate for a critical sized defect) and dense tissue growth over bone ends at defect site. Likewise, experimental samples displayed similar characteristics across all time intervals. All experimental samples had a slight surface defect present (although size decreased as time progressed) and intermedullary canal was continuous throughout entire femoral length. Thus $\mu \mathrm{CT}$ provided a non-destructive technique to view internal composition without compromising sample integrity. Non- destructive microcomputed tomography testing allowed femurs to undergo additional testing, thus maximizing the quantity of data per sample.

In the operated control group, repair was present along the ventral femoral surface. Repair was incomplete (discontinuous intermedullary canal with defect on dorsal femoral surface) and resultant area of repair visually appeared as dense cortical bone in a single slice image. In Figure 3-A, the surface defect can be visualized along with an incomplete intermedullary canal. In the experimental group, significant bone repair was observed. In Figure 3-B, the defect was almost completely repaired with the exception of a small surface disruption and the intermedullary canal has begun to reform. 
In the six month operated control group, the defect was still visible and bone was somewhat deformed (Figure 3-C). In the experimental six month group, repair was significantly improved compared to the operated control group during the same time interval. The intermedullary canal was continuous throughout the femur. A slight remnant of the defect in cortical bone on ventral surface remained. Figure 3-D shows the dorsal plane of the defect completely healed and indistinguishable from surrounding bone.

In the nine month operated control group, cortical bone defined edges of the defect (Figure 3-E) and repair of the intermedullary canal was discontinuous. In the nine month experimental group, repair was essentially complete with only small amounts of defect present; the three dimensional rendering showed the absence of a visually apparent defect (Figure 3-F). Figure 3-G showed the unoperated control femur.

\section{CONCLUSIONS}

The specific conclusions were:

1. The animal evaluations of the composite paste for 3 , 6 \& 9 months examined by undecalcified histology and microCT $(\mu \mathrm{CT})$ demonstrated a high degree of bone ingrowth for the experimental group, compared to samples in the operated control group (defect repaired without paste).

2. The use of $\mu \mathrm{CT}$ as a non-destructive tool to follow bone ingrowth was found to be very valuable. Although we used this technique mainly for qualitative data, the data could be digitized to make more quantitative predictions.

3. Purified crawfish chitosan compounded with calcium sulfate in paste form can be successfully used to deliver osteoblasts that will allow bone growth to occur in defects.

\section{ACKNOWLEDGMENTS}

The authors appreciate the help of Errin Robinson and Alan L. Ogden for surgical assistance, Dollie Smith for growing the osteoblasts and Shelia Rogers for guidance regarding the sectioning and staining of slides. The plates were supplied by Synthes (Paoli, PA).

\section{REFERENCES}

[1] Q. Li, E. T. Dunn, E. W. Grandmasion, M. F. A. Goosen. (1992) Applications and Properties of Chitosan. Applications of Chitin and Chitosan, ed. M. F. A. Goosen. Technomic Publishing Company, Inc.: Lancaster. 3-29.

[2] H. No, S. Meyers, (1995) Preparation and characterization of chitin and chitosan-A review. Journal of Aquatic Food Product Technology. 4(2): 27-52.

[3] H. K. No, S. P. Meyers, (1997) Preparation of chitin and chitosan, in Chitin Handbook, R. A. A. Muzzarelli, Peter, M. G., Editor. $475-489$
[4] S. B. Rao, C. P. Sharma, (1997) Use of chitosan as a biomaterial: Studies on its safety and haemostatic potential. Journal of Biomaterials Research. 34: 21-28.

[5] T. Jiang, C. Pilane, C. (2005) Laurencin, Fabrication of Novel Porous Chitosan Matrices as Scaffolds for Bone Tissue Engineering. Materials Research Society Symposium. 845: 187-192.

[6] Y. Chen, Y. Chung, L. Wang, K. Chen, S. Li, (2002) Antibacterial Properties of Chitosan in Waterborne Pathogens. Journal of Environmental Science and Health. A37 (7): 1379-1390.

[7] Z. Shi, K. Neoh, E. Kang, W. Wang, (2006) Antibacterial and mechanical properties of bone cement impregnated with chitosan nanoparticles. Biomaterials. 27: 2440-2449.

[8] T. Don, C. Chuang, W. Chiu, (2002) Studies on the Degradation Behavior of Chitosan-g-Poly (acrylic acid) Copolymers. Tamkang Journal of Science and Engineering. 5(4): 235-240.

[9] P. VandeVord, H. M., S. DeSilva, L. Mayton, B. Wu, P. Wooley, (2002) Evaluation of the biocompatibility of a chitosan scaffold in mice. Journal of Biomedical Materials Research. 59: 585-590.

[10] Y. Okamoto, R. Yano, K. Miyatake, I. Tomohiro, Y. Shigemasa, S. Minami, (2003) Effects of chitin and chitosan on blood coagulation. Carbohydrate Polymers. 53: 337-342.

[11] M. Jumaa, F. H. Furkert, B. W. Müller, (2002) A new lipid emulsion formulation with high antimicrobial efficacy using chitosan. European Journal of Pharmaceutics and Biopharmaceutics. 53: 115-123.

[12] P. A. Felse, T. Panda, (1999) Studies on applications of chitin and its derivatives. Bioprocess and Biosystems Engineering, 20 (6): 505-512.

[13] T. Chandy, C. P. Sharma, (1990) Chitosan as a Biomaterial. Biomaterials, Artificial Cells and Artificial Organs. 18(1): 1-24.

[14] P. K. Dutta, J. Dutta, M. C. Chattopadhyaya, V. S. Tripathi, (2004) Chitin and Chitosan: Novel Biomaterials Waiting for Future Development. Journal of Polymer Materials. 21(3): 321-333.

[15] S. Mallapragada, B. Narasimhan, (2006) Handbook of Biodegradable Polymeric Materials and their Applications. Vol. 1 \& 2.

[16] A. K. Singla, M. Chawla, (2001) Chitosan: some pharmaceutical and biological aspects-an update. Journal of Pharmacy and Pharmacology. 53(8): 1047-1067.

[17] D. Mukherjee, (2001) Method for producing chitin or chitosan, US patent $6310,188 \mathrm{~B} 1$, Oct. 30 .

[18] T. A. Einhorn, J. M. Lane, A. H. Burstein, C. B. Kopman and V. J. Vigorita, (1984) The healing of segmental defects induced by demineralized bone matrix, J. of Bone and Joint Surgery, 66-A: 274-279.

[19] D. W. Wolff, V. M. Goldberg and S. Stevenson (1994) Histomorphometric analysis of the repair of a segmental diaphyseal defect with ceramic and titanium fiber metal implants: effect of bone marrow. J. of Orthopaedic Research. 12(3): 439-446.

[20] D. P. Mukherjee, A. S. Tunkle, R. A. Roberts, A. Clavenna, S. Rogers, D. Smith, (2003) An Animal Evaluation of a Paste of Chitosan Glutamate and Hydroxyapatite as a Synthetic Bone Graft Material. Journal of Biomedical Material Research, Part B. 67B: 603-609.

[21] T. L. N. Jarvinen, P. Kannus, H. Sievanen, P. Jolma, A. Heinonen, M. Jarvinen, (1998) Randomized controlled study of effects of sudden impact loading on rat femur. Journal of Bone and Mineral Research. 13(9): 1475-1482.

[22] Y. Umemura, T. Ishiko, T. Yamauchi, M. Kurono, S. Mashiko, (1997) Five jumps per day increase bone mass and breaking force in rats. Journal of Bone and Mineral Research. 12(9): 1480-1485. 